\title{
Effets des informations chimiques provenant d'un milieu habité par des congénères sur l'orientation topographique du poisson cavernicole Phreatichthys andruzzii Vinciguerra (Pisces, Cyprinidae).*
}

\author{
R. Berti**, G. Thinès ${ }^{* * *}$ et B. Lefèvre***
}

\author{
SUMMARY
}

Two series of experiments were performed on the oriented locomotor responses of 27 specimens of the blind cave fish Phreatichthys andruzzii from Somalia using a three-compartment choice apparatus. The oriented responses were observed individually from the central compartment towards either of the extreme ones. In one of them, $500 \mathrm{ml}$ water were introduced from either the tank in which the test fish had previously resided with conspecifics (1st series, 46 experiments) or from another tank occupied by unknown conspecifics, the other compartment receiving an equivalent volume of pure water. The two series were performed in random blocks of 6 experiments, the momentary position of the test fish being noted every 30 seconds after an adaptation period of at least 4 hours. Results, analyzed in 9 blocks of 5 minutes show a definite preferential orientation of the fishes for the compartment containing chemical information from both known or unknown conspecifics. This effect is discussed in relation to the ecological conditions in which the species under study lives.

\section{INTRODUCTION}

Le rôle des stimulations sensorielles susceptibles de déterminer l'orientation de la locomotion chez les poissons cavernicoles a été principalement étudié, à l'origine, sous le rapport de la stimulation photique et, secondairement, sous le rapport de

* Recherche effectuée avec l'aide du Centro di Studio per la Faunistica ed Ecologia Tropicali del Consiglio Nazionale delle Ricerche (Dir. Prof. L. Pardi).

** Istituto di Zoologia dell'Università, Via Romana 17, I-50125 Firenze (Italie).

*** Centre Albert Michotte, Biologie du Comportement, Université de Louvain, B-3041 Pellenberg (Belgique). 
la stimulation mécanique. Ces deux types de signaux ne présentent pas une importance de principe égale dans le guidage de la locomotion en raison des conditions écologiques dans lesquelles vivent ces espèces. Les données relativement nombreuses que l'on possède au sujet des effets de la stimulation lumineuse sont difficiles à interpréter du point de vue de l'adaptation biologique, vu qu'elles concernent une modalité sensorielle dont la valeur de survie n'est pas évidente chez les organismes vivant dans un milieu perpétuellement obscur. Les quelques hypothèses que l'on peut émettre à ce sujet ont été discutées dans le travail d'ensemble de Thinès (1969). Plus récemment trois études de Ercolini et Berti $(1975,1977,1978 a)$ ont également abordé le problème de la sensibilité photique chez les poissons cavernicoles somaliens. Les auteurs précités ont du reste principalement envisagé les potentialités réceptrices des poissons étudiés, et n'ont abordé le problème de l'utilité biologique de ces réponses que de façon secondaire. Toutefois, s'il n'est pas exclu qu'une réaction photonégative contribue à maintenir dans une certaine mesure les poissons cavernicoles à l'intérieur de leur biotope obscur et à les soustraire, en principe, grâce à ce mécanisme à la pression sélective élevée du milieu épigé, il y a lieu de remarquer que dans certains types d'habitats, la stimulation lumineuse semble moins déterminante que dans d'autres. En effet, certaines espèces comme Uegitglanis zammaranoi qui vit dans la nappe phréatique somalienne, visitent les zones éclairées artificielles comme les puits, où elles sont apparemment attirées par des signaux chimiques provenant des débris organiques probablement plus nombreux à cet endroit. Cependant, comme l'ont observé Ercolini et Berti (1978), l'Uegitglanis zammaranoi ne visite qu'exceptionnellement les puits pendant le jour, ce qui semble indiquer que l'obscurité favorise l'extension de ces explorations dans les parties épigées artificielles du milieu. Les mêmes auteurs supposent en outre que ces visites nocturnes sont également favorisées par le fait que les milieux extérieurs sont dénués à ces moments, de víbrations mécaniques résultant de la présence humaine. Dans la mesure où cet exemple est représentatif, les comportments observés peuvent être expliqués à partir de deux causes possibles: 1) l'uniformité de l'obscurité pendant la période nocturne qui' rendrait indistinctes les limites de l'habitat et, 2) la présence dans les zones exposées à la lumière pendant la période diurne, de substances chimiques liées à la présence de matières orgañques plus abondantes en ces endroits. Il est difficile dans l'état actuel de nos connaissances, en particulier en raison de notre ignorance des conditions biologiques exactes présentes dans la nappe phréatique, de décider de l'importance relative de ces diverses stimulations 
dans le déclenchement des comportements observés. De toute façon le fait que l'Uegitglanis et d'autres espèces cavernicoles soient observés dans des zones éclairées comme les puits indique à première vue que les réponses chimiques dominent dans certains cas les réponses photonégatives. Ces quelques remarques doivent induire à la prudence lorsque l'on tente d'interpretér les résponses photiques observées en laboratoire, particulièrement en ce qui concerne le signe négatif des réponses.

Quant aux signaux mécaniques, bien qu'ils constituent une source d'information à distance porticulièrement bien adaptée en principe, aux conditions du milieu souterrain obscur, ils ne semblent pas avoir pris une importance particulière dans les aspects constructifs de l'evolution des Vertébrés cavernicoles. Le système latéral des Téléostomes et des Urodèles cavernicoles présente en effet un développement très variable mais guère différent de celui que l'on observe chez les formes épigées (Thinès et Durand, 1973 - Thinès, 1979). Cependant, dans une série d'études consacrées au comportement alimentaire, divers auteurs ont abordé secondairement cette question en raison $\mathrm{du}$ fait que, dans certaines circonstances, les particules alimentaires peuvent être détectées tant par voie mécanique que par voie chimique (Thinès, Soffié et Vandenbussche 1966 - Thinès et Wissocq, 1972 - Thinès et Capon, 1975). Ajoutons que les conditions physiques caractérisant les milieux dans lesquels vivent les espèces cavernicoles peuvent ne pas être favorables à la transmission de signaux mécaniques dans le milieu liquide, même s'il est vrai que ces signaux permettent théoriquement un réperage facile dans l'obscurité.

Dans le cas de la nappe phréatique, il est fort probable que la topographie du milieu est loin d'être uniforme et offre sans doute de nombreux plans de réflexion (roches, failles, etc.) qui peuvent non seulement empêcher une propagation linéaire directe du signal mécanique et causer une déformation consécutive des ondes mécaniques, mais également provoquer un amortissement irrégulier et non homogène des ondes de propagation. Dans ces conditions, si les signaux mécaniques peuvent jouer un certain rôle dans le repérage d'objets et de congénères à courte distance, il semble improbable qu'ils puissent fournir des indices suffisants dans la localisation précise et facile de la source émissive à longue distance, ce qui n'est pas à négliger étant donné les distances considérables couvertes par ces habitats. Dans les milieux karstiques, par ailleurs, les chutes d'eau qui se déversent dans certaines nappes déterminent fort probablement, dans certains cas, un masquage pratiquement permanent des signaux mécaniques utilisables par les poissons. 
On peut conclure de ce qui précède que la stimulation mécanique semble présenter trop d'inconvénients pour garantir, au moins dans les milieux naturels, la transmission précise à distance de toute une série d'informations facilement exploitables par les poissons. Dès lors, sans nier que la transmission de signaux de nature mécanique puisse jouer un rôle important dans la biologie des poissons cavernicoles, il nous semble qu'elle représente à elle seule un moyen relativement peu puissant pour véhiculer les informations nécessaires à l'orientation de ces poissons.

A ce propos, il ne nous semble pas erroné d'avancer l'hypothèse selon laquelle une autre source d'information pourrait intervenir et qui pourrait, selon nous, consister en une transmission d'information par voie chimique. La transmission d'information par des signaux d'un tel type présenterait plusieurs avantages par rapport à la précédente: 1) dans l'espace, la formation de gradients de concentration offre la possibilité de déterminer aisément la localisation de la source émissive et la distance à laquelle elle se trouve, 2) les signaux chimiques bien étalés dans le temps et relativement stables, permettent le transport passif d'informations même à grande distance, sans diminution sensible de leur directionalté et de leur contenu.

L'étude du rôle des signaux chimiques dans le comportement des poissons cavernicoles paraît d'autant plus justifiée, qu'outre l'adéquation de principe déjà signalée de ces signaux aux conditions physiques du milieu souterrain, il s'agit d'un aspect de l'éthologie de ces espèces qui n'a pas été abordé jusqu'à présent de façon systématique. Si ces signaux interviennent en effet dans l'orientation de la locomotion, il doivent probablement revêtir une signification biologique bien définie dans la vie sociale de ces poissons. Cet aspect de la biologie des cavernicoles est fort mal connu, principalement en raison des difficultés que l'on rencontre dans l'observation des comportements des cavernicoles dans leur milieu naturel et de l'impossibilité absolue, jusqu'à présent, de procéder à de telles observations sur les espèces qui vivent dans la nappe phréatique. Ainsi, donc, les faits qui sont discutés dans ce travail débouchent indirectement, à notre sens, sur l'étude éthologique proprement dite des Vertébrés cavernicoles, laquelle soulève de toute évidence des problèmes très ardus comme Thinès (1979) l'a signalé.

Dans le présent travail, nous avons fait l'hypothèse qu'un milieu occupé pendant un certain temps par un groupe de poissons cavernicoles était caractérisé par la présence d'informations chimiques liées à la présence des poissons et susceptibles de guider l'orientation locomotrice de congénères. 


\section{MATERIEL ET METHODE}

Dans la présente recherche, nous avons disposé de 27 poissons recoltés en juin 1978 dans les puits de Bud-Bud (Rép. Dém. Somalienne) et répartis en deux groupes désignés par $a$ et $b$ de 13 et 14 exemplaires respectivement, quelques jours après leur capture. Dès ce moment, et jusqu'au terme de nos expériences, c'est-à-dire pendant une période de 10 mois environ, ils ont été placés dans deux aquariums distincts, désignés par $: A$ et $B$ entre lesquels tout contact direct ou indirect a été soigneusement évité. En dépit du fait que les poissons avaient été réunis en un groupe unique au cours de leur capture, nous avons estimé qu'une telle période suffisait à exclure toute reconnaissance entre les individus des deux groupes, reconnaissance qui serait liée à une éventuelle mémoire des caractéristiques chimiques propres au groupe initial.

Dans nos expériences, les poissons pouvaient s'orienter individuellement à partir de le zone centrale de l'aquarium, vers l'une ou l'autre de ses extrémités, l'une de celles-ci contenant de l'eau dans laquelle avaient séjourné préalablement des congénères, selon le schéma décrit plus loin. Dans ces conditions, on pouvait aisément observer la préférence manifestée par l'animal pour l'une ou l'autre des deux zones, selon la fréquence avec laquelle il visitait l'une ou l'autre de celles-ci. Nous avons effectué deux séries d'expériences: dans la première, nous avons étudié la réaction des poissons aux traces chimiques provenant de leur propre aquarium de stockage, et donc de leur groupe d'appartenance. Dans la seconde, nous avons étudié la réaction des poissons aux traces chimiques provenant de l'aquarium de stockage de l'autre groupe de poissons, qui leur était inconnu. Pour simplifier les choses, nous parlerons d'un schéma $a \times A$ ou $b \times B$ dans le cas de la prémière série, ou d'un schéma $a \times B$ ou $b \times A$ dans le cas de la seconde série, c'està-dire selon que le milieu d'où provenaient les informations chimiques était occupé par un groupe de congénères connus ou non du poisson-test. Les expériences de la prémière série (46 au total) et celles de la seconde série (36 au total) ont été effectuées au cours d'une même période de temps (Mars-Avril 79). Les deux séries ont été effectuées par blocs de 6 expériences en ordre aléatoire, afin de rendre les donnés comparables.

\section{Dispositif expérimental et conduite des expériences.}

Le dispositif expérimental était constitué de deux aquariums de stockage, d'une batterie de 6 aquariums expérimentaux et d'un aquarium contenant de l'eau de réserve. Les deux groupes 
de poissons dont nous disposions ont été placés pendant les six mois avant le début des expériences et durant celles-ci, dans deux aquariums identiques de $100 \mathrm{l}$ de capacité chacun. Les seuls objets présents dans les aquariums étaient les tuyaux de la pompe assurant le filtrage de l'eau.

Afin d'éliminer autant que possible toute turbulence, le tuyau de sortie de la pompe était inséré dans un cylindre en matière plastique de $33.5 \mathrm{~cm}$ de hauteur et de $5 \mathrm{~cm}$ de diamètre, placé verticalement dans un coin de l'aquarium. Ce cylindre était ouvert à son extrémité supérieure, ce qui permettait l'écoulement de l'eau filtrée à travers la pompe. De plus, on prenait la hauteur du cylindre comme référence pour contrôler la baisse du niveau de l'eau consécutive à l'évaporation; celle-ci était alors compensée en adjoignant de l'eau distillée, afin d'éviter une concentration progressive des éléments en solution.

Les aquariums expérimentaux étaient de longs parallélépipèdes en verre collé de $180 \mathrm{~cm}$ de longueur, de $8 \mathrm{~cm}$ de largeur et de $20 \mathrm{~cm}$ de hauteur (Fig. 1). Des glissières appliquées à la surface interne de l'aquarium pouvaient recevoir des cloisons mobiles en matière plastique qui, une fois en place, partageaient l'aquarium en 3 sections de $60 \mathrm{~cm}$ de longueur chacune. Chacune des 3 sections décrites ci-dessus était fermée à sa partie supérieure au moyen d'un couvercle en plexiglas transparent, permettant de réaliser dans l'aquarium un éclairage dépourvu de toute zone d'ombre. Un petit espace entre les trois couvercles permettait de soulever les cloisons de leur siège. Deux supports en matière plastique, placés aux deux extrémités de l'aquarium (Fig. 1-b), pouvaient recevoir deux flaconslaveurs en matière plastique d'une capacité de $500 \mathrm{ml}$ (Fig. 1-a).

Le dispositif était enfin completé par un aquarium de $200 \mathrm{l}$ de capacité utilisé comme réserve d'eau. Cet aquarium était pourvu d'une résistance thermostatique qui permettait d'élevèr très rapidement la température de l'eau, il était également pourvu d'une pompe qui assurait une circulation permanente de l'eau et empêchait la formation de couches d'eau de température différente.

$\mathrm{Au}$ cours de chaque expérience, le poisson qui allait être testé était prélevé de son propre aquarium de stockage et placé individuellement dans la section centrale de l'aquarium expérimental, les cloisons étant en place. Le transfert du poisson était effectué de 4 à 14 heures environ avant le début de l'observation, pour permettre à l'animal de s'adapter au milieu expérimental et, en outre, de surmonter le stress dû au transfert. Après cette période d'adaptation, on plaçait simultanément, à chacune des exrémités de l'aquarium expérimental, les deux flacons-laveurs remplis, l'un avec de l'eau tirée de l'aquarium de stockage d'où provenait le poisson ou de l'aquarium 


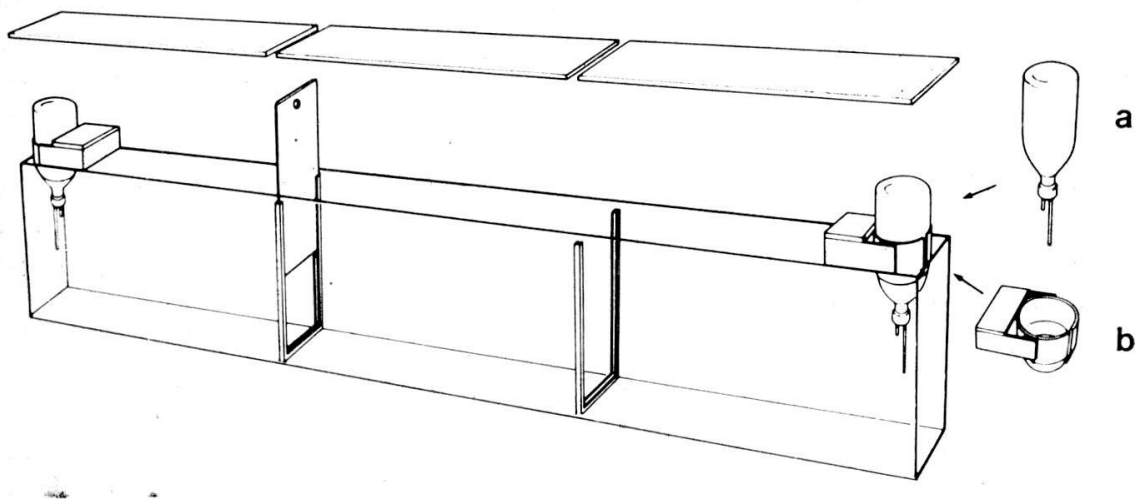

Fig. 1 - Schéma du dispositif expérimental montrant les trois sections de l'aquarium expérimental pourvues de leurs glissières et de leurs couvercles.

a: flacon-laveur

b: support du flacon-laveur

de stockage de l'autre groupe de poissons, l'autre avec de l'eau provenant de la réserve. L'extrémité de l'aquarium à laquelle on devait appliquer le flacon contenant l'eau tirée d'un des deux aquariums de stockage avait été préalablement déterminée en utilisant une table de nombres au hazard de telle façon que ce flacon soit disposé un nombre égal de fois d'un côté et de l'autre soit au cours d'une même séance expérimentale, soit sur l'ensemble des expériences. De la même facon, on a rendu aléatoire la durée de la période d'adaptation qui précédait les expériences.

Les flacons-laveurs étant pourvus de 2 tubes, l'un pour la sortie de l'eau, l'autre pour la rentrée de l'air, se vidaient sans bruit, en 30 secondes environ, après quoi ils étaient retirés. Cinq minutes après l'application des flacons (période indispensable pour permettre une diffusion suffisante de l'eau dans les 2 sections de l'aquarium expérimental), on enlevait les cloisons de séparation et on commençait l'observation. Cette dernière, d'une durée totale de 45 minutes, consistait en un repérage et en un enregistrement, toutes les 30 secondes, de la position du poisson-test dans l'une ou l'autre des 3 sections de l'aquarium expérimental.

Chaque séance expérimentale portait sur un total de 6 expériences. On testait donc 6 poissons: 3 prélevés d'un groupe, 3 de l'autre.

Etant donné le nombre assez limité de poissons dont nous disposions, nous avons été obligés de tester plusieurs fois les 
mêmes individus. Etant donné d'une part qu'il était impossible de marquer, et donc de reconnaître individuellement chaque animal et qu'il était nécessaire d'autre part de soumettre tous les poissons à un nombre comparable de tests en respectant entre 2 tests successifs sur le mème poisson un délai suffisant, les poissons ont été identifiés sur la base de leur taille (grande, moyenne ou petite). Les individus utilisés au cours d'une même séance avaient approximativement la même grandeur. Ce procédé a permis de satisfaire aux exigences méthodologiques qui viennent d'être mentionnées. Les 6 poissons à tester dans chaque séance expérimentale étaient transférés dans les aquariums expérimentaux à la même heure et également replacés à la même heure dans leur aquarium de stockage à la fin de la séance. On procédait ensuite à la vidange et au nettoyage des aquariums expérimentaux. Le remplissage de ces derniers était effectué deux heures environ avant le transfert, dans l'aquarium expérimental même, des poissons à tester dans la séance suivante. L'eau utilisée pour le remplissage des 2 flacons-laveurs présentait des caractéristiques physico-chimiques comparables à l'eau présente dans les aquariums expérimentaux, vu qu'elle était tirée de la même réserve. Dans toutes les expériences, la hauteur de l'eau était de $8 \mathrm{~cm}$ en sorte que le volume d'eau dans chacune des trois sections de l'aquarium expérimental était de $\pm 3,8 \mathrm{l}$. Les aquariums de stockage, les aquariums expérimentaux et le réservoir d'eau se trouvaient dans le local même où s'effectuaient les expériences; celui-ci était thermostatisé de façon que l'eau de tous les aquariums présentait une température de $28.3^{\circ} \mathrm{C}$. Le local était continuellement éclairé par 8 ampoules rouges de 25 Watts chacune, dont le spectre d'émission est donné à la Fig. 2. Les 6 aquariums expérimentaux étaient placés au milieu du local leur grand côté étant perpendiculaire à l'alignement des lampes, chacune d'entre elles surplombant le point central de chaque aquarium expérimental. Cette disposition des sources lumineuses a été adoptée afin d'éviter un éclairage différentiel de part et d'autre des aquariums mêmes, étant donné la photophobie très marquée observée chez le Phreatichthys. Celle-ci est toutefois moins nette pour les grandes longueurs d'onde (Ercolini et Berti, 1975).

Pendant les 60 jours qui ont précédé le début des expériences, les poissons ont été nourris avec de la nourriture sèche pour poissons tropicaux d'eau douce, une fois tous les deux jours au minimum, et une fois tous les quatre jours au maximum. Ce rythme a été maintenu au cours des expériences elles-mêmes à ceci près que les poissons n'étaient plus nourris au cours des 20 heures précédant les séances expérimentales. En outre, le filtrage de l'eau était interrompu avant chaque distribution de nourriture et n'était rétabli qu'après consommation totale 


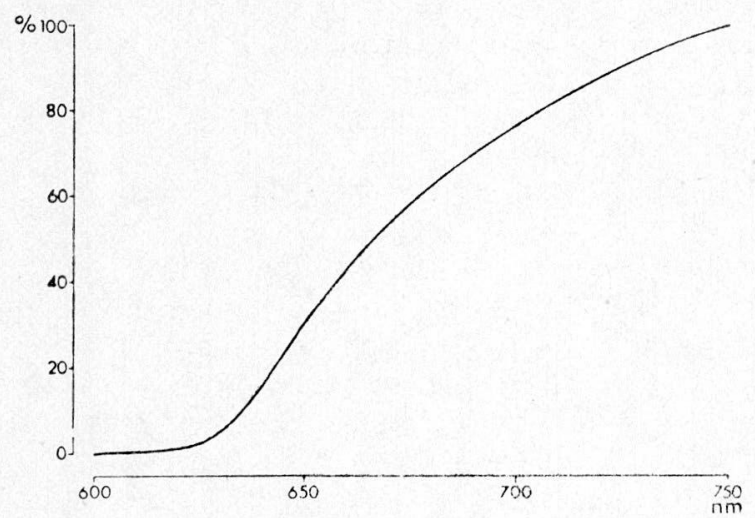

Fig. 2 - Spectre d'émission des sources lumineuses. E'n abscisse: longueur d'onde en $\mathrm{nm}$ En ordonnée: émission relative

des quantités distribuées afin d'éviter que d'éventuelles particules résiduelles ne perturbent la réaction des poissons aux signaux chimiques des congénères. Toutes les manipulations ont été les mêmes pour les deux aquariums. On peut donc considérer qu'avant les expériences et au cours de celles-ci, les deux groupes de poissons $a$ et $b$ ont été placés dans des conditions identiques.

\section{RESULTATS}

Prémière série.

Dans cette série, les informations chimiques provenant de l'aquarium occupé par le groupe $a$ étaient fournies à un poisson-test qui avait lui-même séjourné en $A$ et celles provenant de l'aquarium occupé par le groupe $b$ étaient fournies à un poisson-test qui avait séjourné en $B$ (schéma $a \times A$ ou $b \times B$ mentionné plus haut: 46 expériences). Ces résultats d'ensemble obtenus pour cette série sont donnés à la Fig. 3, où sont représentées les fréquences absolues de présence des poissons-tests dans trois zones de l'aquarium expérimental, telles qu'elles ont été relevées toutes les 30 secondes au cours des 45 minutes d'observations effectuées pour chacune des 46 expériences.

L'examen de cette figure montre à l'évidence que la section de l'aquarium expérimental la plus fréquentée par les poissonstests est celle dans laquelle avait été introduite de l'eau prove- 

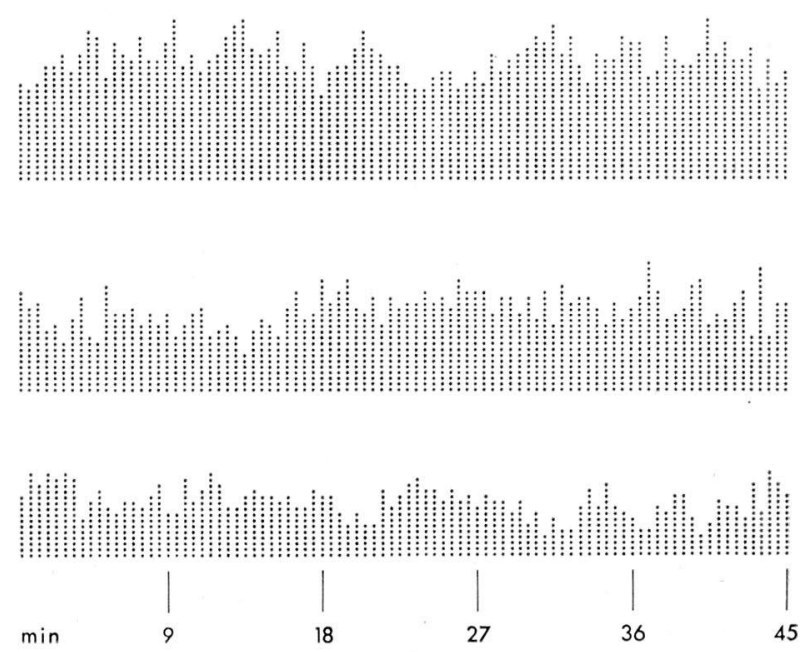

Fig. 3 - Fréquences absolues de présence des poissons-tests dans les trois sections de l'aquarium expérimental (Expériences $a \times A$ et $b \times B$ : congénères connus).

Histogramme supérieur: section extrême contenant les traces chimiques Histogramme central: section centrale

Histogramme inférieur: section extrême dépourvue de traces chimiques En abscisse: durée totale de l'observation (45 minutes; 90 pointages). Chaque point en ordonnée représente un pointage (total par colonne: 46 points)

nant de leur aquarium de stockage. Les données relatives à cette section sont représentées à la partie supérieure de la Fig. 3. La comparaison des fréquences obtenues pour cette section et de celles obtenues pour la section extrême opposée (partie inférieure de la figure) met nettement en évidence la supériorité numérique des premières, et permet de mieux évaluer la préférence manifestée pour la section contenant les traces chimiques sur toute la durée de l'expérience. Quant à la partie centrale de la Fig. 3, elle traduit les fréquences de présence dans la section centrale de l'aquarium expérimental, lesquelles sont dues à la fois à des choix non orientés et a,ux passages obligés du poisson-test lorsqu'il nage d'une section extrême à l'autre. Les données relatives à la zone centrale ne peuvent être comparées, comme telles, à celles des deux zones extrêmes parce que la section centrale de l'aquarium n'est pas équivalente topographiquement aux deux autres: sa géométrie ne comporte en effet pas les limites extrêmes; en outre il s'agit de la section 
dans laquelle le poisson-test séjournait pendant la période d'adaptation d'une durée de 4 à 14 heures avant le début des observations (voir p. 108). La section centrale était donc, contrairement aux deux autres, bien connue du poisson-test lorsqu'il pouvait effectuer son choix au moment où l'on retirait les parois de séparation. Pour ces raisons, les données de la zone centrale ne sont pas prises en considération dans l'analyse statistique des résultats. En ce qui concerne cette dernière, les 46 expériences ont été rangées en trois classes de fréquences (Positive, Neutre et Négative) selon que, sur la durée totale de chaque expérience ( 45 minutes, 90 données), le nombre de pointages effectués dans la section contenant l'information chimique, était supérieur, égal ou inférieur au nombre de pointages effectués dans la section extrême opposée de l'aquarium. Les différences relevées entre les effectifs des classes Positive et Négative ont été traitées par le $\chi^{2}$ (test à un échantillon, Siegel, 1956). Le même traitement a été appliqué au déroulement temporel des expériences. Chacune d'entre elles a été répartie en 5 périodes de 9 minutes qui ont été elles-mêmes rangées dans les trois classes de fréquences précitées. Les résultats de ces analyses sont donnés au Tableau 1.

Tab. 1 - Analyse statistique des résultats obtenus dans les expériences $a x A$ et $b \times B$ (congénères connus): 46 expériences.

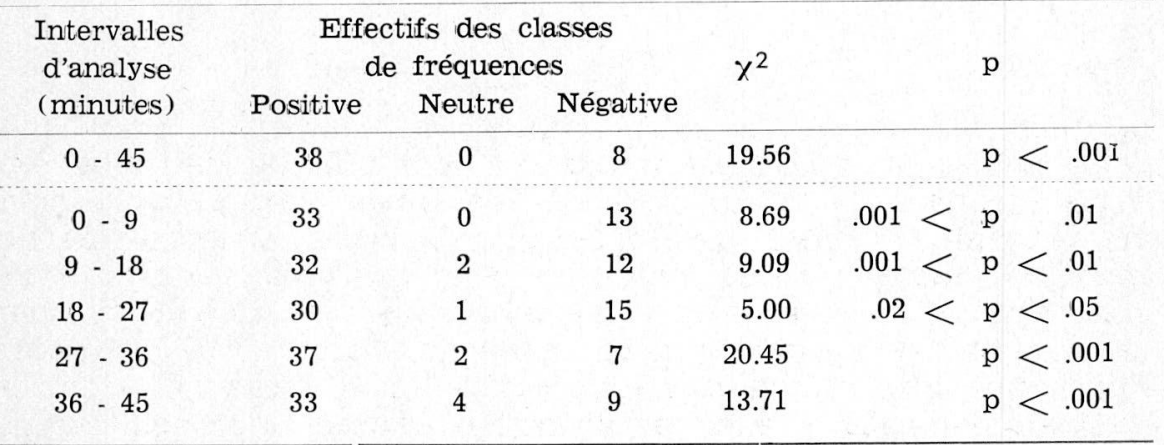

Tant dans le cas des classes de fréquences considérées sur la durée totale de l'expérience que dans le cas des classes de fréquences établies par période, les différences sont significatives.

L'évolution des différences au cours des 5 périodes peut être caractérisée comme suit: 
1) Le choix des poissons en faveur de la zone contenant une information chimique provenant de congénères connus, est très net au cours des 2 premières périodes, c'est-à-dire jusqu'à 18 minutes après le début des observations.

2) On note une fluctuation dans la préférence des poissons au cours de la 3ème période, c'est-à-dire de 18 à 27 minutes après le début des observations.

3) Le choix initial des poissons en faveur de la zone contenant des traces chimiques de congénères connus est confirmé et même renforcé au cours des 2 dernières périodes (de $27 \mathrm{mi}$ nutes à 45 minutes après le début des expériences).

\section{Seconde Série.}

Dans la seconde série, les informations provenant de l'aquarium occupé par le groupe $a$ étaient fournies à un poissontest qui avait séjourné en $B$ et celles provenant de l'aquarium occupé par le groupe $b$ étaient fournies à un poisson-test qui avait séjourné en $A$ (schéma $a \times B$ et $b \times A$ mentionné plus haut:
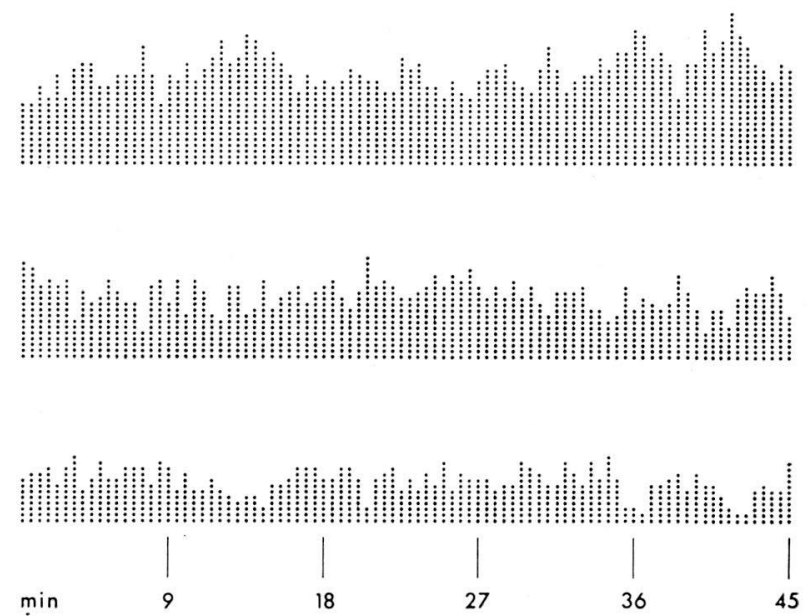

Fig. 4 . Fréquences absolues de présence des poissons-tests dans les trois sections de l'aquarium expérimental (Expériences $b x A$ et $a x B$ : congénères inconnus).

Histogramme supérieur: section extrême contenant les traces chimiques Histogramme central: section centrale

Histogramme inférieur: section extrême dépourvue de traces chimiques En abscisse: durée totale de l'observation ( 45 minutes; 90 pointages). Chaque point en ordonnée représente un pointage (total par colonne: 36 points), 
36 expériences). Les résultats d'ensemble obtenus pour cette série sont donnés à la Fig. 4, où, les fréquences absolues sont représentées comme à la Fig. 3. Ici encore, la zone la plus fréquentée par les poissons est celle dans laquelle on avait indroduit de l'eau provenant de l'aquarium de stockage, ce milieu étant, rappelons-le, inconnu du poisson-test. L'analyse statistique, effectuée selon la méthode citée plus haut, met en évidence des différences significatives tant entre les classes de fréquences considérées sur la durée totale de l'expérience qu'entre les classes établies par période. Toutefois, le niveau de signification est légèrement inférieur en ce qui concerne la durée totale $(.001<\mathrm{p}<.01$ contre $\mathrm{p}<.001$ dans la première série). En outre, l'évolution des différences au cours des 5 périodes est tout à fait comparable à celle qui avait été mise en évidence dans la première série, à ceci près qu'au cours de la première période, les poissons ne manifestent aucune préférence significative pour la zone contenant une information provenant de congénères inconnus.

Tab. 2 - Analyse statistique des résultats obtenus dans les expériences $b \times A$ et $a \times B$ (congénères inconnus): 36 expériences.

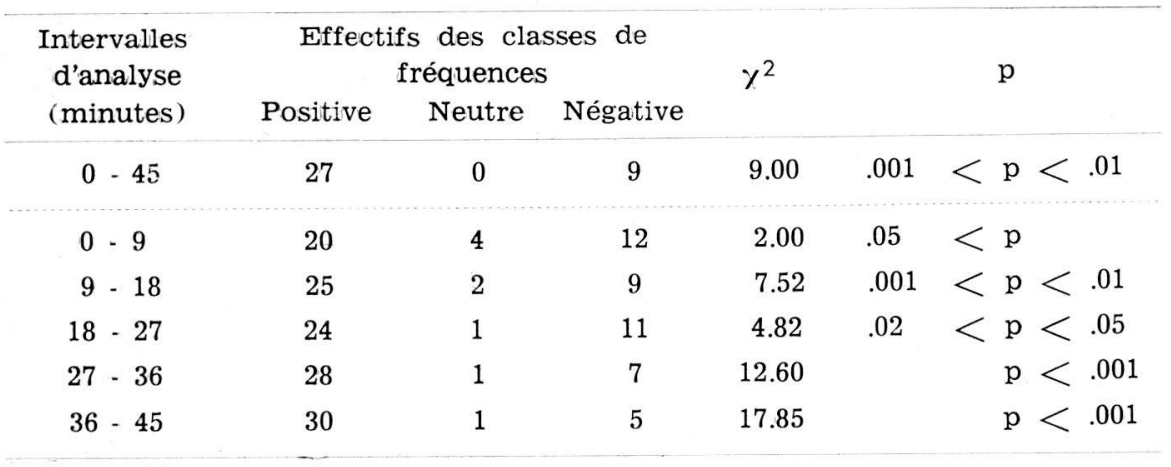

\section{DISCUSSION ET CONCLUSIONS}

Il importe de souligner avant toute chose le fait que les résultats obtenus dans le schéma $(a \times A ; b \times B)$ et dans le schéma $(a \times B ; b \times A)$ vont dans le même sens: qu'il s'agisse de traces chimiques de congénères connus ou inconnus, les poissons ont tendance à s'orienter préférentiellement en direction de celleci. Cependant, malgré leur convergence d'ensemble, les résultats des deux séries présentent certaines différences caractéristiques dans leur évolution temporelle. Dans le cas d'une trace chimique provenant d'un milieu connu (1ère série), on obser- 
ve dès les premières minutes de l'expérience une polarisation de la locomotion vers la zone qui contient cette information. Cette orientation initiale est néanmoins suivie d'une légère diminution du phénomène qui se traduit par une intensification de l'exploration dans diverses directions. Au terme de celle-ci (c'est-à-dire au cours des 18 dernières minutes de l'expérience), la polarisation vers la zone initialement choisie réapparaît et semble définitivement établie. La même évolution s'observe dans le cas d'une trace chimique provenant de congénère inconnus (2de série), avec toutefois cette différence importante qu'il n'y a dans ce cas aucune polarisation initiale nette avant la 9ème minute.

On peut donc supposer ou bien que contrairement aux traces chimiques de congénères connus, ces traces de congénères inconnus ne sont identifiées qu'après un certain temps de latence, ou bien que, tout en étant identifiées comme signalant la présence de congénères, elles informent l'animal-test de la présence d'individus étrangers à son groupe. Ceci expliquerait qu'il y ait attraction (20 cas positifs contre 12 négatifs et 1 neutres au cours des premières 9 minutes) sans que celle-ci atteigne toutefois immédiatement un niveau d'intensité comparable à celui qui s'observe pour l'odeur de congénères connus.

Bien que nos expériences aient été réalisées dans des conditions très différentes de celles du milieu naturel et après une longue période de captivité, les réactions observées nous semblent extrêmement intéressantes dans le contexte éco-éthologique. Les Phreatichthys seraient apparemment capables d'utiliser des signaux chimiques émis pour gagner ou regagner une zone du milieu occupé par des congénères après s'en être éloignés activement ou en avoir été éloignés passivement, comme p. ex. sous l'action d'un courant. Ceci semble d'autant plus probable qu'il s'agit de populations monospécifiques (dans le cas contraire, en effet, d'autres espèces de poissons cavernicoles auraient été capturées dans les puits au même titre que les Phreatichthys) et que, de ce fait, toute trace chimique signale nécessairement la présence de congénères. Au vu des résultats obtenus, on peut supposer qu'en raison des effets de la monospécificité, les traces chimiques de l'espèce constituent des signaux capables d'orienter la locomotion d'un individu et qu'en outre une certaine reconnaissance du groupe semble intervenir sur la base des mêmes informations.

\section{REMERCIEMENTS}

Les auteurs remercient le Professeur A. Ercolini, de l'Institut de Zoologie de l'Université de Florence, qui a mis à leur disposition le matériel zoologique, a participé à l'élaboration du programme de recherche et a procédé à 
le lecture critique du manuscrit. Ils remerciement également Mlle M. Jankow. ska qui leur a apporté une aide appréciable dans la réalisation des expériences.

\section{RESUME}

Deux séries d'expériences out été réalisées sur les réponses locomotrices d'orientation de 27 specimens du poisson cavernicole aveugle Phreatichthys andruzzii Vinciguerra originaire de la Somalie, dans un appareil à choix à trois compartiments. L'orientation locomotrice des poissons a été relevée individuellement à partir du compartiment central vers l'un des deux compartiments extrêmes. Dans l'un de ceux-ci on introduisait $500 \mathrm{ml}$ d'eau provenant, soit de l'aquarium de stockage où le poisson-test avait séjourné antérieurement avec des congénères (1ère série, 46 expériences), soit d'un aquarium de stockage dans lequel se trouvaient des congénères inconnus (2de série, 36 expériences), l'autre compartiment recevant un volume équivalent d'eau pure. Les deux séries ont été effectuées par blocs de 6 séances expérimentales de 45 minutes en ordre aléatoire, la position momentanée du poissontest étant relevée toutes les 30 secondes après une période d'adaptation de 4 heures minimum. Les résultats, analysés par tranches de 9 périodes de 5 minutes, mettent en évidence une orientation préférentielle très nette des poissons pour le compartiment contenant une information chimique de congénères tant connus qu'inconnus. Cet effet est discuté en relation avec les conditions écologiques dans lesquelles vit l'espèce étudiée.

\section{TRAVAUX CITES}

ERCOLINI, A. \& R. BERTI 1975. Light sensitivity experiments and morphology studies of the blind phreatic fish Phreatichtys andruzzii Vinciguerra from Somalia. Monitore zool. ital. (N.S.) Suppl. 6: 29-43.

ERCOLINI, A. \& R. BERTI 1977. Morphology and response to light of Uegitglanis zammaranoi Gianferrari, anophthalmic phreatic fish from Somalia. Monitore zool. ital. (N.S.) Suppl. 9: 183-199.

ERCOLINI, A. \& R. BERTI 1978a. Morphology and response to light of Barbopsis devecchii Di Caporiacco (Cyprinidae), microphtalmic phreatic fish from Somalia. Monitore zool. ital. (N.S.) Suppl. 10: 299-314.

ERCOLINI, A. \& R. BERTI 1978b. Communication personnelle.

SIEGEL, S. 1956. Nonparametric statistics for the behavioral sciences. International Student Edition. Tokyo: Kogakusña Co. Ltd. XVII + 312 pp.

THINES, G. 1969. L'évolution régressive des Poissons cavernicoles et abyssaux. Paris: Masson et Cie. Editeurs 354 pp.

THINES, G. 1979. L'éthologie des Téléostomes cavernicoles: Hypothèses sur l'évolution des comportements. Bull. Acad. Royale Belg., Classe des Sciences, 5ème série, 65 (9): 387-399.

THINES, G. \& D. CAPON 1975. L'aveuglement expérimental de Poissons épigés et la cécité génétique des Poissons cavernicoles: effets sur le comportement alimentaire. Spelunca (Mém.) 8: 209-228.

THINES, G. \& J. DURAND 1973. Connaissances actuelles sur l'apparat sensoriel de la ligne latérale chez les Vertébrés cavernicoles aquatiques. Annls Spéléol. 28 (2): 271-282.

THINES, G., M. SOFFIE \& E. VANDENBUSSCHE 1966. Analyse du comportement alimentaire du poissons cavernicole aveugle Anoptichthys Gen. et d'hybrides $\mathrm{F}_{1}$ (Astyanax $\mathrm{X}$ Anoptichthys) et $\mathrm{F}_{2}$. Int. J. Speleol. 2:437-448.

THINES, G. \& N. WISSOCQ 1972. Etude comparée du comportement alimentaire de deux poissons cavernicoles (Anoptichthys jordani Hubbs et Innes et Caecobarbus geertsi Blgr.). Int. J. Speleol. 4:139-169. 